\title{
Cordonnier Grade 1 Fungal Complication, Superficial Candida Infection
}

National Cancer Institute

\section{Source}

National Cancer Institute. Cordonnier Grade 1 Fungal Complication, Superficial Candida

Infection. NCl Thesaurus. Code C138298.

Any superficial Candida infection. 